\title{
BMJ Open Barriers to patient enrolment in phase III cancer clinical trials: interviews with clinicians and pharmaceutical industry representatives
}

\author{
Aniek Dane (D) , ${ }^{1}$ Soedaba Ashraf, ${ }^{2}$ James Timmis, ${ }^{3,4}$ Monique Bos, ${ }^{5}$ \\ Carin Uyl-de Groot, ${ }^{6}$ P Hugo M van der Kuy (D) ${ }^{1}$
}

To cite: Dane A, Ashraf S, Timmis J, et al. Barriers to patient enrolment in phase III cancer clinical trials: interviews with clinicians and pharmaceutical industry representatives. BMJ Open 2022;12:e055165. doi:10.1136/ bmjopen-2021-055165

- Prepublication history and additional supplemental material for this paper are available online. To view these files, please visit the journal online (http://dx.doi.org/10.1136/ bmjopen-2021-055165).

Received 07 July 2021 Accepted 28 January 2022

Check for updates

(C) Author(s) (or their employer(s)) 2022. Re-use permitted under CC BY-NC. No commercial re-use. See rights and permissions. Published by BMJ.

For numbered affiliations see end of article.

Correspondence to

Drs Aniek Dane;

a.dane@erasmusmc.nl

\section{ABSTRACT}

Objectives Phase III cancer clinical trials are expensive and time-consuming phases in drug development. Effective patient enrolment can reduce delays and save costs, offering patients an opportunity to benefit from innovative treatments. However, the current evidence base does not fully explain the persistence of barriers to patient enrolment in phase III cancer clinical trials. The aim was to explore clinicians' and pharmaceutical representatives' views on these barriers.

Design A qualitative study was performed. In-depth information was collected from 15 experts in the field of oncology clinical trials, in particular clinical oncologists acting as principal investigators (PIs) and clinical research associates. By means of semistructured interviews, based on a questionnaire derived from our newly developed conceptual framework, they were asked to identify barriers to patient enrolment they had experienced and comment on barriers identified in literature.

Findings Existing knowledge on barriers to patient enrolment was confirmed by all interviewees. Two new key barriers to patient enrolment were identified, that is, insufficient attention to the importance of clinical trial-based research in medical training and a trust gap between Pls and pharmaceutical representatives. A third important barrier was increasingly narrow patient inclusion criteria.

Conclusions The success rate of patient enrolment in phase III cancer clinical trials highly depends on the clinicians' willingness to take part in clinical trials. Raising awareness of the importance of clinical trials in medical training and among practising oncologists is recommended. Furthermore, to reduce barriers to patient enrolment, it is essential that both clinicians and pharmaceutical representatives acknowledge each other's expertise, become acquainted with each other's procedures and regulations, and work on building trust relationships. Finally, in accordance with our key findings, we propose to add two new barriers to our newly developed conceptual framework; insufficient attention to clinical trial research in medical training and trust gap.

\section{BACKGROUND}

Cancer drug development is a long, expensive and risky process, with phase III
Strengths and limitations of this study

- This study takes the perspectives of principal investigators, clinicians as well as pharmaceutical industry representatives on barriers to patient enrolment.

- To guide the questionnaire, a new framework was developed to identify barriers to patient enrolment.

- The qualitative study design based on barriers identified in literature, gave room to participants to complement these barriers with their own experience.

- Limitations include-due to the first outbreak of COVID-19 - that merely recently retired principal investigators were interviewed.

- Data validity may be impacted as most of the interviewees were found via the same network of doctors which might have triggered self-selection bias.

clinical trials being among the most expensive phases. ${ }^{12}$ Nowadays, with rapidly rising costs of cancer drugs, governments, healthcare providers and insurers are increasingly concerned about affordable and sustainable patient access to cancer treatment. ${ }^{34}$ Although it is not exactly clear what the costs of drug development, ${ }^{56}$ especially phase III trials, are-estimates range from US $\$ 9 \mathrm{~m}$ to US $\$ 33 \mathrm{~m}^{178}$ - there is little dispute that these costs are mainly caused by the efforts needed to enrol large groups of trial participants. ${ }^{9}$ Various studies have shown that a substantial number of clinical trial sites fail to recruit the required number of patients timely, resulting in wasted resources due to costly delays or even study abandonment. ${ }^{10} 11$ Only $2 \%-5 \%$ of adult cancer patients are successfully enrolled in clinical trials. ${ }^{12}$ A systematic review by Bentley presented that almost $24 \%$ of the included articles made a recommendation concerning improving patient accrual to reduce trial costs. ${ }^{13}$ Therefore, effective patient enrolment is crucial. ${ }^{14}$ However, research is often focused on patient-related 
barriers during the enrolment process and on strategies how to improve the inclusion of eligible patients. ${ }^{15}$ Limited evidence exists on patient enrolment barriers that are physician- or pharmaceutical industry related. We found some existing knowledge on provider-related barriers that influence patient enrolment in a model presented by Howerton et al, such as physician' awareness of and attitude towards clinical trials. ${ }^{16}$ Likewise, Grunfeld et al, Frank, and Caulfield have identified some barriers to patient enrolment from a pharmaceutical industry perspective, such as recruitment costs, recruitment strategy and complex protocols concerning inclusion and exclusion criteria. ${ }^{17-19}$

To further explore the barriers to patient enrolment in phase III cancer clinical trials and by such improve pertinent recruitment practice, our aim was to explore these barriers from the perspectives of principal investigators (PIs), clinicians and pharmaceutical representatives.

\section{METHODS}

Following the Standards for Reporting Qualitative Research (SRQR) guidelines a qualitative study was performed by means of semistructured one-on-one interviews (online supplemental appendix 1) ${ }^{20}$ This research design provided us with the means to examine the topic more in-depth than a quantitative study would have allowed. ${ }^{21} 22$

Existing knowledge of patient enrolment barriers to clinical trials as perceived by clinicians and the pharmaceutical industry (online supplemental appendix 2) ${ }^{17-19}$ was used to develop a conceptual framework to guide our interviews. After completing the questionnaire and the interview guide, we tested the questionnaire on one PI, to check for clarity and internal validity.

In-depth information for this study was collected from various experts in the field of cancer clinical trials $(n>10)$, namely pharmaceutical representatives working as clinical research associates (CRAs), and clinical oncologists of whom most were PIs (table 1). All potential participants were situated in the Netherlands. The initial five participants were selected from the professional network of the authors, after which the sample was expanded via personal requests of the initial participants in their own professional network. Potential participants were personally invited by email, which also included detailed information on the aim of the study, its design and informed consent.

The interviews were conducted in Dutch by SA. Each interview was started by confirming informed consent of the interviewee, by guaranteeing confidentiality of the personal data and by explaining the interviewee's right on privacy and right to withdraw from the study. ${ }^{22}$ Furthermore, we guaranteed all participants anonymity, moreover when permission was given to quote them in this study. All interview questions were open-ended to provide interviewees with flexibility in answering the questions based on their personal perspectives. We used probing questions to ensure that all relevant topics were covered, yet leaving room for more explanation. ${ }^{22}$

Interviews were audiorecorded, transcribed and afterwards immediately deleted. Before data was analysed, the interviewees were offered the opportunity to review a summary of their interview transcript, of which none of them made use of. To prevent translation bias verbatim translation of the transcripts to English was performed. Personal data were secured in a safe environment of SURFdrive to which access was limited. To further ensure safety of data storage, we made sure the research data management plan followed the appropriate scientific European Union (EU) guidelines.

Track of barriers mentioned in each interview was kept to determine when data saturation had been reached. ${ }^{23}$ Data were analysed by SA via thematic analysis to identify broad patterns and themes, starting from a reflection on existing barriers and adding new variants (and themes) via an inductive approach. ${ }^{24}$ Data were coded using ATLAS.ti V.8 coding software. On a regular basis, we discussed analytical procedures, as well as the interpretation of preliminary results and the presentation of the results.

\section{Patient and public involvement}

We did not involve patients or general public in this study, for the reason that the scope of our study was on the initiating actors of sponsor-initiated cancer clinical trials.

\section{RESULTS}

We invited 22 potential participants in March 2020, of whom 15 were interviewed online in March and April 2020. Each interview lasted between 40 and $60 \mathrm{~min}$. Data saturation was reached in interview 13 (figure 1), identifying 67 unique concepts (online supplemental appendix $3)$.

\section{Barriers-to-patient-enrolment framework}

Our developed framework 'Barriers to patient enrolment according to clinical research centres and pharmaceutical industry' (figure 2) was composed of patient-related, physician-related and industry-related barriers. We used these subsequent barriers to guide our results. An overview of the key results is provided in table 2 .

\section{Patient-related barriers}

Interviewees expressed that important patient-related barriers were awareness and motivation. Almost all of them mentioned a lack of patient awareness of the availability of clinical trials. Regarding motivation, some CRAs stated that patient motivation or willingness to participate in clinical trials largely depended on clinicians' communication skills. All interviewees considered that lack of patient awareness and motivation might be linked to physician and industry-related barriers.

\section{Physician-related barriers}

Regarding physician-related barriers, all interviewees mentioned awareness, attitude and motivation. According 
Table 1 Overview specification of the interviewees

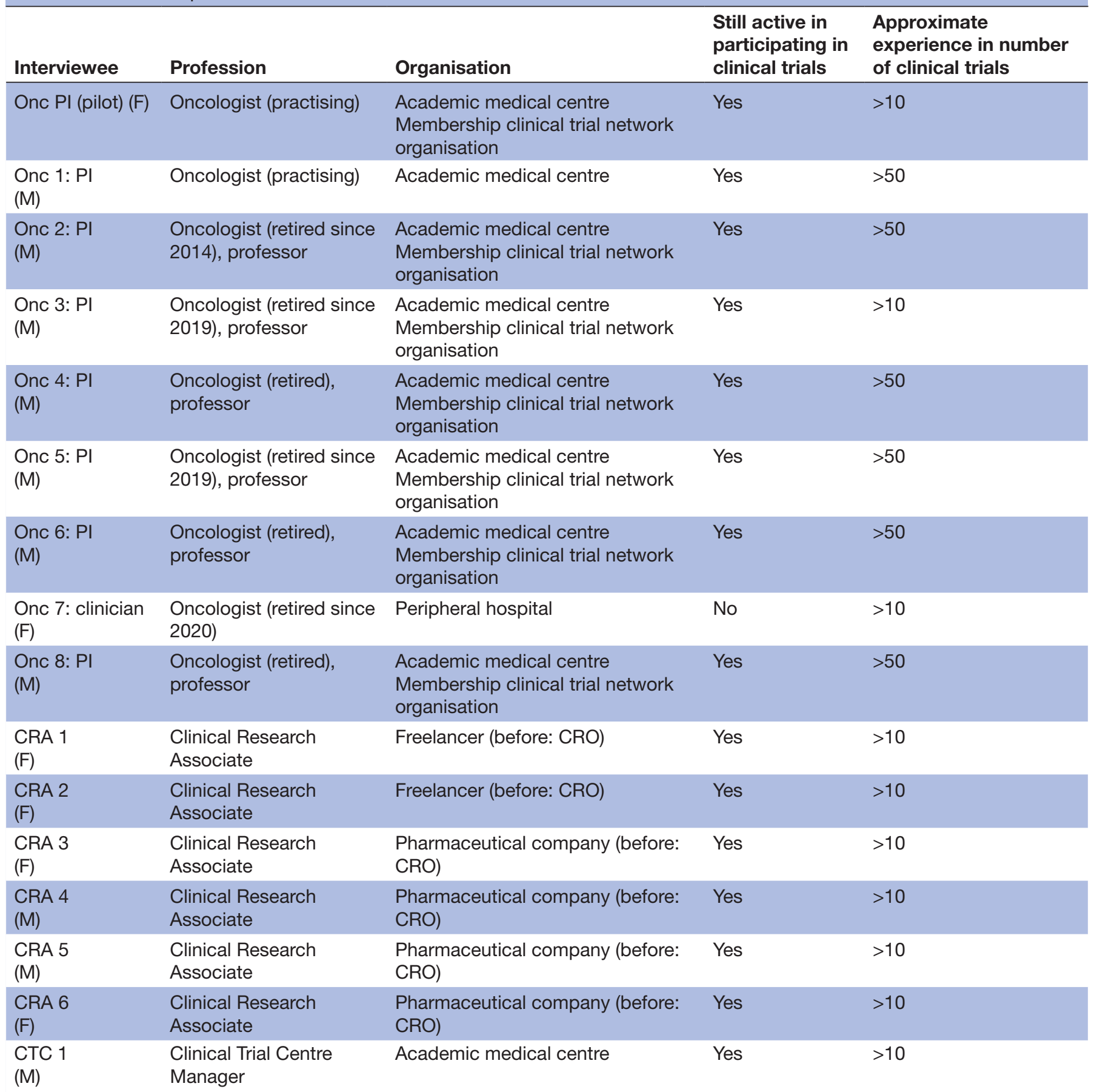

CRA, clinical research associate; CRO, clinical research organisation; CTC, clinical trial centre; F, female; M, male; Onc, oncologist; PI, principal investigator.

to most PIs, a lack of awareness of the importance of clinical trials was caused by insufficient attention to this kind of research in medical training. A component of awareness, mentioned by several CRAs, was that clinicians would forget to participate in clinical trials due to lack of time and busy schedules. Furthermore, they would prioritise on delivering healthcare instead of participating in clinical research.
PI 1: 'Current education for trainee clinicians is very much focused on providing standard care. We need more clinicians to be educating in the clinical research field, but clinicians and scientists do not always think the same way.'

As to the second barrier, all PIs agreed that clinicians' attitudes towards clinical trials were negative if they expected that a trial under consideration would not fulfil 


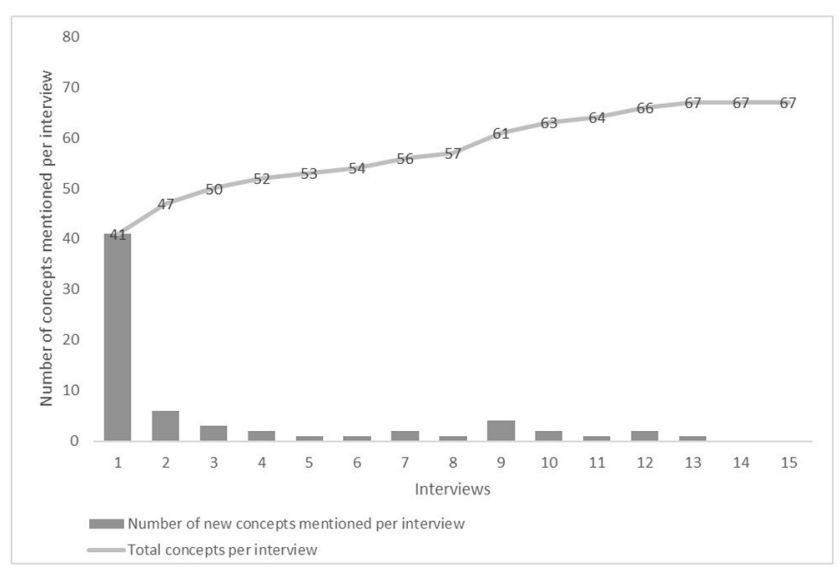

Figure 1 Data saturation curve.

patient needs. Consequently, most PIs stated that patient motivation and therefore recruitment would probably remain low if clinicians did not make use of their patientdoctor relationship and emphasise the benefits of participating in clinical trials to their patients, for reasons of additional medical attention, chance of successful treatment, reimbursement of travel costs, and contribution to a possible treatment for future patients. Conversely, most CRAs expressed that a positive attitude might be hindered by animosity regarding trial benefits between involved clinicians, resulting in clinicians withholding patients from a particular trial.

The last mentioned barrier was physician motivation. According to all interviewees, willingness to participate was less likely if clinicians were not (1) acknowledged in publications, (2) given any monetary incentives, (3) convinced of the relevance of the research question, or most importantly, if they did not intrinsically believe in the positive effects of a clinical trial, and the benefits to their patients. However, some CRAs emphasised that sometimes patient recruitment stopped prematurely once the clinicians had received payment. Financial incentives were also mentioned by some PIs, who stated that it might be financially more attractive for clinicians, and to a larger extent hospitals, to provide standard care rather than being involved in clinical research, because they were not compensated for the additional time invested in clinical research.

\section{Industry-related barriers}

All interviewees agreed that industry-related barriers were mostly linked to clinical trial costs, regulatory and administrative procedures, and collaboration between the pharmaceutical industry and clinicians.

With reference to the first barrier-clinical trial costsall interviewees emphasised high staff and protocol amendment costs. Comparing time spent on regular consultations with that of patient recruitment, most oncologists specified that the latter takes three to four times longer. Although some costs were being covered by the study sponsor, if the budget was insufficient for hiring support research personnel, the study could be delayed. CRAs mentioned that, once the study had started, costs increased largely if alterations in inclusion criteria (due to lack of sufficient patient enrolment) were necessary. These alterations necessitated other or additional requests for amendments to the Medical Ethical Committee, causing further study delay.

The second mentioned barrier-regulatory and administrative procedures-consists of three components, namely (1) protocol complexity of clinical trials and subsequent administrative burden, (2) inclusion and exclusion criteria for patient selection, and (3) extensive informed consent regulations. All interviewees agreed

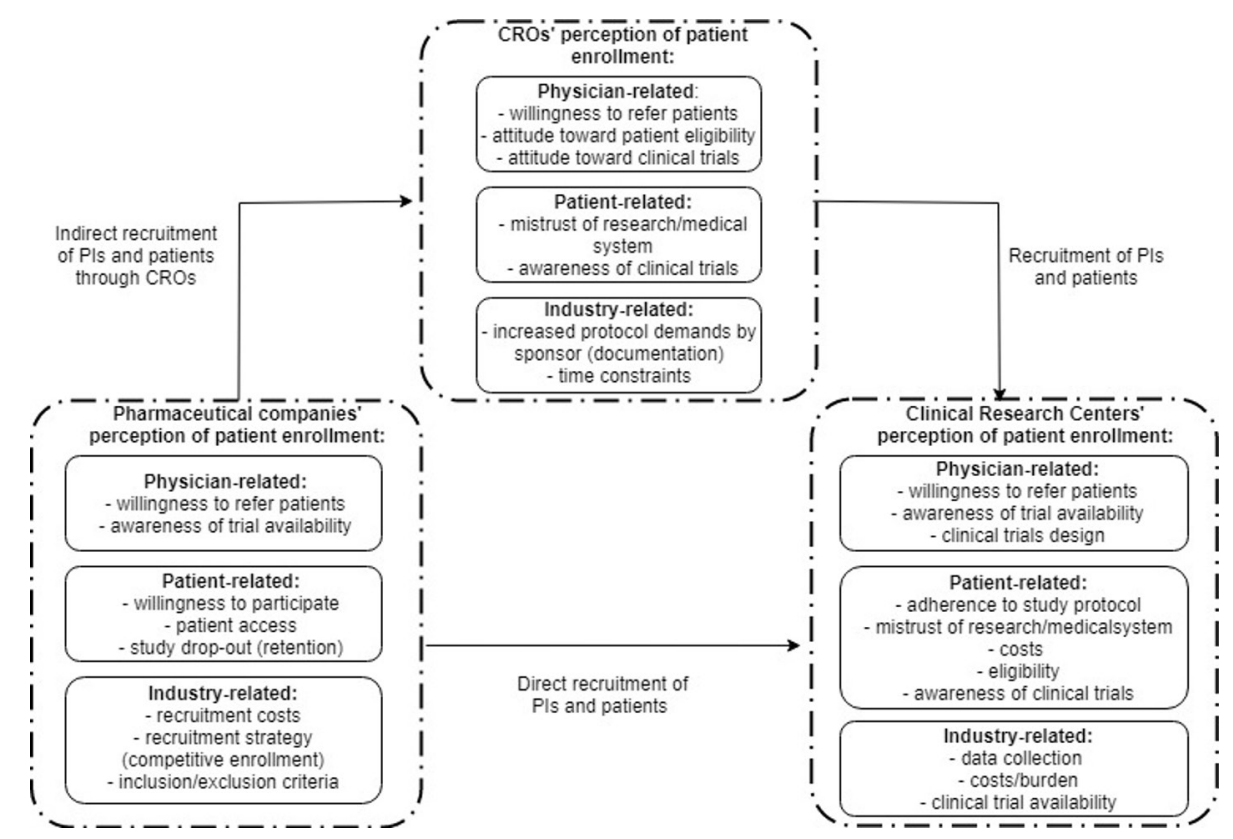

Figure 2 Framework of barriers to patient enrolment according to clinical research centres and pharmaceutical industry. CRO, clinical research organisation; Pls, principal investigators. 
Table 2 Summary key results

\begin{tabular}{|c|c|c|}
\hline Patient-related barriers & PIs/clinicians & CRAs \\
\hline $\begin{array}{l}\text { Lack of patient awareness and } \\
\text { motivation }\end{array}$ & $\begin{array}{l}\text { No knowledge of the availability of trials } \\
\text { Motivation depended on industry or clinician }\end{array}$ & $\begin{array}{l}\text { No knowledge of the availability of } \\
\text { trials } \\
\text { Motivation depended on industry or } \\
\text { clinician }\end{array}$ \\
\hline Physician-related barriers & PIs/clinicians & CRAs \\
\hline Lack of physician awareness & $\begin{array}{l}\text { Due to insufficient medical training to the } \\
\text { importance of clinical trials }\end{array}$ & Due to lack of time and busy schedules \\
\hline Negative physician attitude & $\begin{array}{l}\text { Not convinced whether trials fulfil patients' } \\
\text { needs clinicians do not emphasise benefits } \\
\text { to patients }\end{array}$ & $\begin{array}{l}\text { Clinicians have insufficient } \\
\text { communication skills animosity } \\
\text { between clinicians }\end{array}$ \\
\hline Lack of physician motivation & $\begin{array}{l}\text { Due to lack of acknowledgement in } \\
\text { publications, less monetary incentives, not } \\
\text { convinced of relevance or no intrinsic believe } \\
\text { in positive effects }\end{array}$ & Emphasise on monetary incentives \\
\hline $\begin{array}{l}\text { Regulatory/administrative procedures: } \\
\text { 1. Protocol complexity } \\
\text { 2. Inclusion and exclusion criteria for } \\
\text { patient selection } \\
\text { 3. Extensive informed consent } \\
\text { regulations }\end{array}$ & $\begin{array}{l}\text { Increased complexity of study protocol, } \\
\text { commanded by the study sponsor criteria } \\
\text { have become stricter over the years, } \\
\text { leading to undesirable outcome effects } \\
\text { complex forms that demotivate patients } \\
\text { for pharmaceutical companies to cover up } \\
\text { liability towards regulatory authorities }\end{array}$ & $\begin{array}{l}\text { Increased complexity of study protocol, } \\
\text { demanded by EMA criteria have } \\
\text { become stricter over the years, leading } \\
\text { to increased study duration and study } \\
\text { delay complex forms legally required } \\
\text { by EMA }\end{array}$ \\
\hline $\begin{array}{l}\text { Collaboration between clinicians and } \\
\text { pharmaceutical representatives }\end{array}$ & $\begin{array}{l}\text { Lack of trust due pharmaceutical } \\
\text { representatives overruling clinicians' } \\
\text { expertise and limiting their autonomy no } \\
\text { guarantee on actual participation extensive } \\
\text { and therefore demotivating time pressure }\end{array}$ & $\begin{array}{l}\text { Lack of trust due to mismatch in } \\
\text { expectations, often on estimates on } \\
\text { the number of eligible patients, causing } \\
\text { study delay and additional costs }\end{array}$ \\
\hline
\end{tabular}

CRA, clinical research associate; EMA, European Medicines Agency; PI, principal investigator.

that the increased complexity of study protocols, as demanded by study sponsors-generally pharmaceutical companies-caused additional burden on the clinical research staff and patients, specifically regarding administrative work. On this, most oncologists highlighted the importance of data managers and research nurses to help them keep on track. Most CRAs acknowledged that this burden was caused by protocol complexity, due to the European Medicines Agency's (EMA) requirements regarding protocol development.

CRA 3: 'I know how hard it can be to meet protocol requirements and document every sigh of a patient. But it is possible that the inspection service of the EMA pays an unexpected visit to the center and wants to check if everything is documented. They are very strict. If it is not documented, it did not happen. This puts the study in danger of being delayed or even terminated.'

According to the majority of the PIs, the second component-patient inclusion and exclusion criteria-had become increasingly stricter over the years, which resulted in a very small percentage of otherwise potentially eligible patients being enrolled in trials.

CRA 3: 'In oncology trials one strives to have the healthiest cancer patient in the trial, but there is not such a thing as a healthy cancer patient.'

Ultimately, in terms of efficacy and safety, this might lead to inaccurate outcome estimates and adverse events in the real-world population. Also, CRAs recognised increasingly narrow inclusion criteria as a barrier to patient enrolment and, therefore, as a concern for study duration and corresponding avoidable delays.

Most interviewees agreed that the third component-extensive informed consent regulations-created another barrier for patients to participate in a particular trial. Most PIs indicated that these forms, written in formal language with legal formulations, demotivate and even frighten patients. Subsequently, they suspected that sometimes the pharmaceutical companies extended informed consent forms to cover their liability towards 
regulatory authorities, without considering the burden this places on patients. Similarly, most CRAs stated that the extensiveness of the informed consent form could not be avoided because of the legal requirements of the EMA. Finally, all interviewees mentioned barriers to collaboration between the pharmaceutical industry and clinicians. Most PIs felt that industry set their own strict protocols, thereby effectively overruling the formers' clinical expertise, limiting autonomy, and taking control over patient care delivery. Moreover, some PIs stated that when pharmaceutical companies contracted PIs to manage patient recruitment for trials, they tended to stick to a list of PIs with whom they had already successfully collaborated. This made it more difficult for PIs who were not on said list to be involved in new studies.

PI 1: 'You may see it as 'Tinder', where the industry sponsor swipes left or right. Once in a while, the sponsor plans a 'date' with a doctor to discuss how many patients he/she can include, what quality of data will be provided, how reliable the data will be etc.'

Furthermore, oncologists mentioned that the sponsor requested them beforehand to fill out many forms with confidential information, both on their patients as on their clinical practice. This was considered time consuming, without any guarantee to eventually participate in the study. By having to recruit patients within the time frame given by the sponsoring pharmaceutical company, they experienced considerable additional time pressure, subsequently resulting in clinicians being demotivated to put effort into patient recruitment. Ultimately, all these practices gave rise to feelings of mistrust towards the pharmaceutical industry.

Correspondingly, from the CRAs' perspectives a trust gap was highlighted. The sponsor expected a certain number of patients to be recruited, as 'promised' by the PI. However, according to two CRAs, clinicians often quoted estimates-based on the average number of patients who visited them per year-without considering whether those patients would de facto qualify for inclusion. Hence, if a study sponsor had no insight into the potential number of recruitable patients, the sponsor had to simply trust PIs estimates. If they incorrectly estimated the number of eligible patients, this increased time pressure and possibly led to study delays, causing tension between PIs and industry sponsors. Hence, to control the enrolment process industry tended to stick to its protocol, leaving clinicians little autonomy.

\section{DISCUSSION}

Our aim was to explore clinicians' and pharmaceutical representatives' views of the key barriers to the patient enrolment process of phase III oncology clinical trials in an effort to further improve pertinent recruitment practice.

Our study resulted in three key findings contributing to patient enrolment barriers:
1. Insufficient attention to the importance of clinical research in medical training of clinicians.

2. A trust gap between clinicians and CRAs during the enrolment phase, which is likely caused by industry overruling clinicians' autonomy and expertise and, likewise, clinicians not being explicit on the number of eligible patients vis-à-vis study sponsors.

3. Increasingly narrow patient inclusion criteria and more complex enrolment procedures over the years.

Our first key finding is confirmed by other studies, although this barrier was not mentioned in the models we used in this study. According to the Institute of Medicine, ${ }^{25}$ over the years there seems to be a decrease in motivation among healthcare professionals to pursue a career as PI. According to Mentz et al this might be explained by the considerably growing workload burden for PIs over time and, in contrast, the diminishing rewards of being a PI. ${ }^{26}$ Furthermore, Saleh et al state that with clinical trials becoming more complex, learning pertinent skills on the job is not sufficient. There is a dire need for a structured training and support system at the institutional level such as medical faculties. ${ }^{27}$ As a consequence, the widening separation between clinical research and healthcare delivery is a growing concern for gathering new knowledge. ${ }^{28}$ Therefore, a possible explanation for this key finding might be that we interviewed oncologists of whom most were affiliated with a university medical centre and hence, by default, were more interested in clinical research than the average practising oncologist.

Regarding our second key finding-trust issues between PIs and CRAs-remarkably, according to our used conceptual framework, trust was originally only linked to patientrelated barriers. However, a trust gap between clinicians and pharmaceutical companies was found by Fisher in 2008 , in which institutionalised mistrust was the result of pharmaceutical companies trying to (1) protect their proprietary information from clinicians who conduct their studies and (2) find a way to ensure patients' compliance to study protocol and therefore exert control over their activities. According to Fisher, since study sponsors-most often pharmaceutical companies—by default do not conduct the studies for which they write protocols, have limited interactions with clinicians and none with patients, a model of trust seems impossible. ${ }^{29}$ The results in our study provided by the CRAs reflect this previous study solidly and are apparently still current practice.

Although our third key finding-(increasingly narrow) patient inclusion criteria-is present in our framework, it reflects existing evidence that strict criteria to patient eligibility are currently becoming a crucial barrier to patient enrolment, mainly due to growing concerns of clinicians who face increasing difficulty in converting clinical trial research outcomes, which are based on treatment effects on only eligible patients, to daily practice and their regular-real world-patient population. ${ }^{30-32}$ Also, relaxing inclusion criteria would probably lead to a decrease in trial $\operatorname{costs}^{33}$ and regarding effective design and implementation of clinical trials, Umscheid et al 
stated the importance of clinicians and pharmaceutical industry partnering with the regulatory bodies. ${ }^{1}$ This key finding shows that this concern remains very important for both the pharmaceutical industry and clinicians.

In overall contrast to our model, respondents did not mention trial availability as physician-related barrier. An explanation might be that most interviewed PIs were united in the Dutch Haemato-Oncology Foundation for Adults, which is very much involved in tracking and publishing clinical trial studies and in supporting PIs during trials, such as regular assessments.

Finally, although our results indicate the power of existing knowledge-most already identified barriers were mentioned in this study-we propose to add two new barriers to our newly developed conceptual framework; insufficient attention to the importance of clinical trial research in medical training should be added to physician-related barriers, and the component trust gap should be added to both physician-related and industryrelated barriers. Both are of importance to consider in future enrolling processes.

There were some limitations associated with our study. First, due to unforeseeable circumstances caused by the outbreak of COVID-19 in March 2020 at the onset of this study, we considered it not appropriate to involve practising clinicians, therefore we invited recently retired oncologists from the authors' networks. At a later stage, we interviewed one practising oncologist who confirmed the identified barriers. Second, due to the small sample size consisting of merely haemato-oncologists our results are, potentially, context-dependent. Third, data validity was impacted, as most of the interviewees were found via the same network of doctors which might have triggered self-selection bias. Moreover, due to a lack of available data on the views of general hospital-based practising clinicians the results cannot confirm whether they perceive the barriers the same way as retired PIs and university hospital-based practising clinicians would.

Future research should focus on better understanding the determinants of the barriers we identified and how to overcome them, in particular relating to the trust gap between clinicians and pharmaceutical industry. Insights into this barrier might also contribute to other key barriers. We also suggest investigating barriers to patient enrolment, perceived by oncologists practising in university hospitals vs oncologists practising in general hospitals, since studies are often coordinated by oncologists-PIsin university hospitals, but most of the enrolment efforts are placed on practising clinicians in general hospitals.

\section{CONCLUSION}

We conclude that the success rate of patient enrolment in oncology clinical trials is highly dependent on clinicians' willingness to participate. We recommend to raise awareness of the importance of clinical trials in medical training of future doctors as well as in further training education of practising oncologists. More oncologists participating in clinical trials might increase the likelihood of convincing patients to enrol in clinical trials, which might result in a more rapid onset of clinical trials. Moreover, it is in the patient's best interest for clinicians to not only provide standard care, but also to make sure they are involved in innovative therapies in a timely manner. However, in order to reduce barriers to patient enrolment, and ultimately reduce delays and save costs, it is crucial that both clinicians and pharmaceutical companies work together on bridging trust gaps by first and foremost becoming more acquainted with each other's procedures and regulations. A common ground might be found by joining forces with the regulatory authorities and facilitate conversations around re-evaluation of patient inclusion protocols. ${ }^{34}$

\section{Author affiliations}

${ }^{1}$ Clinical Pharmacy, Erasmus MC, Rotterdam, The Netherlands

${ }^{2}$ Vrije Universiteit Amsterdam, Amsterdam, The Netherlands

${ }^{3}$ Faculty of Science, Vrije Universiteit Amsterdam, Amsterdam, The Netherlands

${ }^{4}$ Institute for Nursing Science, Faculty of Medicine, University of Freiburg, Freiburg im Breisgau, Germany

${ }^{5}$ Internal Oncology, Erasmus MC Cancer Centre, Rotterdam, The Netherlands ${ }^{6}$ Erasmus School of Health Policy \& Management, Erasmus Universiteit Rotterdam, Rotterdam, The Netherlands

\section{Twitter P Hugo M van der Kuy @clinicalrules}

Acknowledgements The authors wish to thank all participants in this study for their disinterested and valuable contribution.

Contributors AD, SA and JT contributed to study design, data acquisition, data analysis, data interpretation, manuscript preparation. MB, CU-dG, HvdK contributed to study design, data interpretation, manuscript preparation. $A D$ acted as guarantor.

Funding The authors have not declared a specific grant for this research from any funding agency in the public, commercial or not-for-profit sectors.

Competing interests None declared.

Patient consent for publication Not applicable.

Ethics approval The study has not been reviewed by an Ethic Committee. Participants gave informed consent to participate in the study before taking part.

Provenance and peer review Not commissioned; externally peer reviewed.

Data availability statement Data are available on reasonable request. All data relevant to the study are included in the article or uploaded as online supplemental information. All interviews have been recorded and removed after transcribing. Transcripts are secured safely. Participants have been guaranteed anonymity. The relevant data are revealed in the manuscript and in appendix 3 which is the codebook used for analysing the transcriptions.

Supplemental material This content has been supplied by the author(s). It has not been vetted by BMJ Publishing Group Limited (BMJ) and may not have been peer-reviewed. Any opinions or recommendations discussed are solely those of the author(s) and are not endorsed by BMJ. BMJ disclaims all liability and responsibility arising from any reliance placed on the content. Where the content includes any translated material, BMJ does not warrant the accuracy and reliability of the translations (including but not limited to local regulations, clinical guidelines, terminology, drug names and drug dosages), and is not responsible for any error and/or omissions arising from translation and adaptation or otherwise.

Open access This is an open access article distributed in accordance with the Creative Commons Attribution Non Commercial (CC BY-NC 4.0) license, which permits others to distribute, remix, adapt, build upon this work non-commercially, and license their derivative works on different terms, provided the original work is properly cited, appropriate credit is given, any changes made indicated, and the use is non-commercial. See: http://creativecommons.org/licenses/by-nc/4.0/.

ORCID iDs

Aniek Dane http://orcid.org/0000-0001-6371-9248 
P Hugo M van der Kuy http://orcid.org/0000-0002-7128-8801

\section{REFERENCES}

1 Umscheid CA, Margolis DJ, Grossman CE. Key concepts of clinical trials: a narrative review. Postgrad Med 2011;123:194-204.

2 PhRMA. Phrma membership survey 2020. Washington DC: Pharmaceutical Research and Manufacturers of America, 2020.

3 Sleijfer S, Verweij J. Health policy: affordability of drugs used in oncology health care. Nat Rev Clin Oncol 2016;13:331-2.

4 Prasad V, De Jesús K, Mailankody S. The high price of anticancer drugs: origins, implications, barriers, solutions. Nat Rev Clin Oncol 2017:14:381-90.

5 DiMasi JA, Grabowski HG, Hansen RW. Innovation in the pharmaceutical industry: New estimates of R\&D costs. J Health Econ 2016;47:20-33.

6 Prasad V, Mailankody S. Research and development spending to bring a single cancer drug to market and revenues after approval. JAMA Intern Med 2017; 177:1569-75.

7 Moore TJ, Zhang H, Anderson G, et al. Estimated costs of pivotal trials for novel therapeutic agents Approved by the US food and drug administration, 2015-2016. JAMA Intern Med 2018;178:1451-7.

8 Sertkaya A, Wong $\mathrm{H}-\mathrm{H}$, Jessup A, et al. Key cost drivers of pharmaceutical clinical trials in the United States. Clin Trials 2016;13:117-26.

9 Getz KA, Kaitin KI. Open innovation: the new face of pharmaceutical research and development. Expert Rev Clin Pharmacol 2012;5:481-3.

10 Drennan KB. Patient recruitment: the costly and growing bottleneck in drug development. Drug Discov Today 2002;7:167-70.

11 Ray K, Tosti B. Transforming patient recruitment through patient and site engagement. IQVIA, 2017.

12 Fouad MN, Lee JY, Catalano PJ, et al. Enrollment of patients with lung and colorectal cancers onto clinical trials. J Oncol Pract 2013;9:e40-7.

13 Bentley C, Cressman S, van der Hoek K, et al. Conducting clinical trials-costs, impacts, and the value of clinical trials networks: a scoping review. Clin Trials 2019;16:183-93.

14 Gupta A, Calfas KJ, Marshall SJ, et al. Clinical trial management of participant recruitment, enrollment, engagement, and retention in the smart study using a marketing and information technology (MARKIT) model. Contemp Clin Trials 2015;42:185-95.

15 Unger JM, Cook E, Tai E, et al. The role of clinical trial participation in cancer research: barriers, evidence, and strategies. Am Soc Clin Oncol Educ Book 2016;35:185-98.

16 Howerton MW, Gibbons MC, Baffi CR, et al. Provider roles in the recruitment of underrepresented populations to cancer clinical trials. Cancer 2007;109:465-76.

17 Grunfeld E, Zitzelsberger L, Coristine M, et al. Barriers and facilitators to enrollment in cancer clinical trials: qualitative study of the perspectives of clinical research associates. Cancer 2002;95:1577-83.

18 Frank G. Current challenges in clinical trial patient recruitment and enrolment. SoCRA Source 2004;2:30-8.

19 Caulfield T. Legal and ethical issues associated with patient recruitment in clinical trials: the case of competitive enrolment. Health Law Rev 2005;13:58-61.

20 O'Brien BC, Harris IB, Beckman TJ, et al. Standards for reporting qualitative research: a synthesis of recommendations. Acad Med 2014;89:1245-51.

21 Britten N. Qualitative interviews in medical research. BMJ 1995;311:251-3.

22 Yeo A, Legard R, Keegan J. In-depth interviews. In: Ritchie J, Lewis J, eds. Qualitative research practice: a guide for social science students and researchers. London: Sage Publications, 2014: 177-210.

23 Palinkas LA, Horwitz SM, Green CA, et al. Purposeful sampling for qualitative data collection and analysis in mixed method implementation research. Adm Policy Ment Health 2015;42:533-44.

24 Braun V, Clarke V. Using thematic analysis in psychology. Qual Res Psychol 2006;3:77-101.

25 National Academic Press (US). Transforming Clinical Research in the United States: Challenges and Opportunities: Workshop Summary. Forum on Drug Discovery, Development, and Translation, 2010.

26 Mentz RJ, Peterson ED. Site principal Investigators in multicenter clinical trials: appropriately recognizing key contributors. Circulation 2017;135:1185-7.

27 Saleh M, Naik G. So you want to be a principal investigator. J Oncol Pract 2018;14:e384-92.

28 Califf RM, Filerman GL, Murray RK. The clinical trials enterprise in the United States: a call for disruptive innovation. IOM forum on drug discovery, development, and translation. Washington DC: Institute of Medicine of the National Acadamies, 2012.

29 Fisher JA. Institutional mistrust in the organization of pharmaceutical clinical trials. Med Health Care Philos 2008;11:403-13.

30 Al-Baimani K, Jonker H, Zhang T. Are clinical trial eligibility criteria an accurate reflection of a real world population of advanced lung cancer patients? J Thorac Oncol 2015;10:S368-9.

31 Shah JJ, Abonour R, Gasparetto C, et al. Analysis of common eligibility criteria of randomized controlled trials in newly diagnosed multiple myeloma patients and extrapolating outcomes. Clin Lymphoma Myeloma Leuk 2017;17:575-83.

32 Klausen TW, Gregersen H, Abildgaard N. The majority of newly diagnosed myeloma patients do not fulfil the inclusion criteria in clinical phase III trials. HemaSphere. Herlev, Denmark: Department of Hematology, Herlev Hospital, 2018: 603-4.

33 Fogel DB. Factors associated with clinical trials that fail and opportunities for improving the likelihood of success: a review. Contemp Clin Trials Commun 2018;11:156-64.

34 Ledford $\mathrm{H}$. Cancer researchers push to relax rules for clinical trials. Nature 2018;556:12-13 